Urologe 2015 · 54:1079-1080

DOI 10.1007/s00120-015-3919-8

Online publiziert: 1. August 2015

(c) Springer-Verlag Berlin Heidelberg 2015

\author{
S. Roth ${ }^{1} \cdot$ A. Schroeder ${ }^{2}$ W. Bühmann ${ }^{3}$ \\ ${ }^{1}$ Klinik für Urologie und Kinderurologie, HELIOS Klinikum Wuppertal, \\ Lehrstuhl für Urologie der Universität Witten/Herdecke, Wuppertal \\ ${ }^{2}$ Berufsverband der Deutschen Urologen e.V., Neumünster \\ ${ }^{3}$ Facharzt für Urologie - Andrologie - Medizinische Tumortherapie - Qualitätsmanagement, \\ Pressesprecher des Berufsverbandes der Deutschen Urologen e.V., Sylt OT Keitum
}

\title{
Berufspolitik = mehr Medizin wagen!
}

Einmal im Jahr,

liebe Kolleginnen und Kollegen,

rücken wir unter Federführung unserer beiden Präsidenten statt eines wissenschaftlichen Schwerpunktes die Berufspolitik ins Leitthema. Warum eigentlich? Wir möchten zeigen, dass die Bedingungen, unter denen wir in Klinik und Praxis arbeiten müssen, um weiterhin angemessen unsere Patientinnen und Patienten behandeln zu können, immer mehr unter unärztlichem Diktat von Ökonomie und Politik leiden. Die beste Berufspolitik wäre die, die sich selbst überflüssig macht. Aber: die Verhältnisse, sie sind nicht so.

Heute bedarf es hochkompetenter ärztlicher Profis, die sowohl in der Patientenversorgung wie auch in den komplexen Prozessen patienten- und arztfremder Steuerungssysteme zuhause sind, um den häufig menschenfeindlichen Ideen profitorientierter Ökonomen als derzeitige "Dominatoren“ des Gesundheitssystems Paroli bieten zu können. Natürlich sind wir nicht unschuldig daran - schließlich haben wir geduldet, dass uns das Primat ärztlicher Entscheidungen als Referenzgröße für erfolgreiches Handeln zugunsten der uns anvertrauten Kranken entzogen wurde. Heute wird Medizin nicht dem Bedarf der Kranken, sondern dem Bedarf der Ökonomen und Aktionäre angepasst - welch Armutszeugnis einer hochkultivierten und wohlhabenden Gesellschaft!

So fächern wir für Sie wiederum das Spektrum unserer berufspolitischen Arbeit auf, um transparent zu machen, wie der durch fach- und sachferne Akteure verursachte Schaden begrenzt werden kann. Deutlich wird die zunehmende Komplexi- tät durch steigende Integration von Juristen und deren Sachverstand - immerhin drei rechtsgelehrte Autoren haben wir eingeladen: mit „Kooperation zwischen Vertragsarzt und Krankenhaus - vor- und nachstationäre Leistungen im Sinne des $\$ 115 \mathrm{a}$ SGB V" beschäftigt sich Christian Gerdts, Fachanwalt für Medizinrecht aus Kiel. Seine Kollegin Sarah Gersch aus Hamburg nimmt sich des Themas „, Renaissance des Honorararztes?" an, einer Variante als Erfindung aus der Zeit des neuen Ärztemangels. Den juristischen Reigen beschließt Frank Schramm, BDU-Justitiar aus Kiel, in Koautorenschaft von Urologe Holger Uhthoff aus Speyer, Sprecher des BDU-Hauptausschusses, mit Abwehrstrategien gegen die skurrilen Regularien zur neuen KV-Regresswelle mit der Zielgruppe zirkumzidierender Urologen, die sich nicht der sinnfreien Pflicht zur histologischen Untersuchung gesunder Vorhäute oder gar der Forderung nach pädophilienaher Fotodokumentation vorher/nachher unterworfen haben: „Neue Probleme mit der Zirkumzision".

Lars Lindemann, Geschäftsführer des Spitzenverbandes der Fachärzte (SpiFa), stellt diese neue, aus der „Potsdamer Runde“ entstandene Kraft als Gesamtvertretung der Facharztgruppen vor in deren engsten Führungszirkel BDU-Präsident Axel Schroeder als 2. Vorsitzender kürzlich gewählt wurde: „Aus Protest entstanden, dem Konsens verpflichtet und durchsetzungsstark".

Dann kommen Ärzte zu Wort: Axel Heidenreich aus Aachen beschreibt mit seinen Koautoren den von ihm geleiteten neuen BDU-Arbeitskreis , Angestellte Ärzte" als Kristallisationsorgan aller nicht frei- 
beruflichen Urologen vom Assistenzarzt in Praxis, MVZ oder Klinik bis zum Ordinarius - angesichts des Nachwuchsproblems eine bedeutende Kraft zur zukunftsorientierten Entwicklung der Urologie.

Die „Ambulante Spezialfachärztliche Versorgung“ als dem Wort gleiche sperrige Konstruktion zur Überwindung der Sektorengrenzen ambulant/stationär bei der Behandlung schwerer Erkrankungen durchleuchtet deren Mitbegründer Andreas Köhler, bis zum letzten Jahresbeginn Vorstandsvorsitzender der Kassenärztlichen Bundesvereinigung und jetziger Ehrenpräsident des SpiFa.

Als Pionier und seit Jahrzehnten aktiver Protagonist schreibt erneut BDUEhrenpräsident und Bundesvorsitzender der Belegärzte Klaus Schalkhäuser Politik und Kostenträgern das weltweit populäre, in Deutschland immer noch ein Schattendasein fristende Belegarztsystem als ideale Verzahnung der beiden Sektoren ins Stammbuch der zu fördernden Konzepte: „Renaissance oder Untergang des Belegarztwesens?"

Für offenen Umgang mit ärztlichen Fehlern im Sinne eines Kulturwandels zur Stärkung der Patientensicherheit plädiert zum Schluss Wolfgang Bühmann, BDUPressesprecher und Initiator des BDUProjekts „CIRS Urologie“ als internetbasiertes, anonymes, sanktionsfreies und selbstlehrendes Fehlermeldesystem.

Das in diesem Heft dokumentierte breite Spektrum berufspolitischer Initiativen soll jedoch nicht durch Absorption ärztlicher Energien davon ablenken, unsere Kraft am stärksten unserer größten Leidenschaft zu widmen: „Mehr Medizin wagen!“

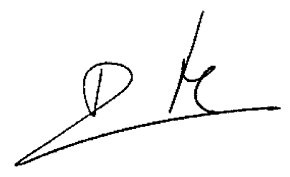

S. Roth

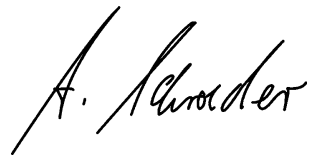

A. Schroeder

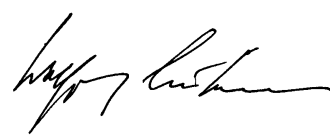

W. Bühmann

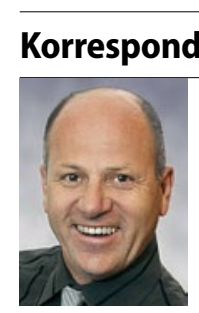

Prof. Dr. S. Roth

Klinik für Urologie und

Kinderurologie, HELIOS

Klinikum Wuppertal, Lehrstuhl

für Urologie der Universität

Witten/Herdecke

Heusnerstraße 40,

42283 Wuppertal

stephan.roth@helios-kliniken.de

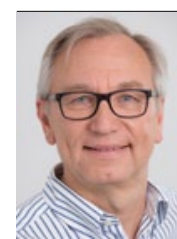

Dr. A. Schroeder

Berufsverband der Deutschen

Urologen e.V.

Haart 87-89,

24534 Neumünster

praesident@bdu-urologie.de

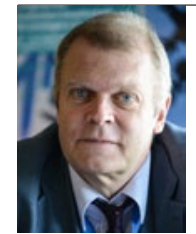

Dr. W. Bühmann

Facharzt für Urologie -

Andrologie - Medizinische

Tumortherapie -

Qualitätsmanagement,

Pressesprecher des

Berufsverbandes der

Deutschen Urologen e.V.

Keitumer Süderstraße 33c, 25980 Sylt OT Keitum post@urologie-sylt.de

Interessenkonflikt. S. Roth, A.. Schroeder u. W. Bühmann geben an, dass kein Interessenkonflikt besteht.

\section{Sicherheit von Medizin- produkten - Neueste Entwicklungen}

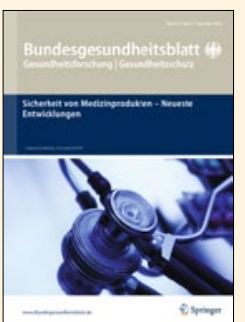

Medizinprodukte leisten einen wichtigen Beitrag zur Prävention, Diagnose und Therapie von Krankheiten. Gleichzeitig stehen Medizinprodukte in

einem Spannungsfeld: Forschung und Entwicklung schreiten schnell voran, minimalinvasive Operationsverfahren ermöglichen schonendere Interventionen, intelligente Implantate unterstützen Körperfunktionen und bieten neue, medizinisch vielversprechende Behandlungsmöglichkeiten. In der Zeitschrift Bundesgesundheitsblatt erschien der Schwerpunkt "Sicherheit von Medizinprodukten - Neueste Entwicklungen" (Ausgabe 12/2014) mit den folgenden Beiträgen zu den wichtigsten Aspekten des Themas:

- Medizinprodukte

- Erfahrungen und Empfehlungen des BfArM zur Genehmigung klinischer Prüfungen von Medizinprodukten und Bewertung schwerwiegender unerwünschter Ereignisse (SAE)

- Register als Werkzeug für mehr Endoprothesensicherheit

- Zukunftstrend "Medical Apps"

Bestellen Sie diese Ausgabe zum Preis von 16,- EUR zzgl. Versandkosten bei Springer Customer Service Center Kundenservice Zeitschriften

Haberstr. 7, 69126 Heidelberg

Tel.: +49 6221-345-4303

Fax: +49 6221-345-4229

E-Mail: leserservice@springer.com

Suchen Sie noch mehr zum Thema? Mit e.Med, dem Online-Paket von Springer Medizin, können Sie schnell und komfortabel in über 500 medizinischen Fachzeitschriften recherchieren.

Weitere Infos unter springermedizin.de/eMed. 